\title{
A NEW CLOUD-EDGE-TERMINAL RESOURCES COLLABORATIVE SCHEDULING FRAMEWORK FOR MULTI-LEVEL VISUALIZATION TASKS OF LARGE-SCALE SPATIO-TEMPORAL DATA
}

\author{
X.M. Li ${ }^{\text {a, }}$, W.X. Wang a, S.J. Tang a, J.Z. Xia ${ }^{\text {a }}$, Z.G. Zhao ${ }^{\text {a }}$, Y. Li ${ }^{\text {a }}$, Y. Zheng a , R. Z.Guo ${ }^{\text {a }}$ \\ ${ }^{\text {a }}$ Research Institute for Smart Cities \& Shenzhen Key Laboratory of Spatial Information Smart Sensing and Services, School of \\ Architecture and Urban Planning, Shenzhen University, Shenzhen, PR China - lixming@ szu.edu.cn; wangwx@ szu.edu.cn; \\ shengjuntang@szu.edu.cn; xiajizhe@szu.edu.cn; zhaozgrisc@szu.edu.cn; liyou@ szu.edu.cn; zhengye@ szu.edu.cn; \\ guorz@szu.edu.cn
}

KEY WORDS: Multi-modal spatio-temporal data, Cloud-edge-terminal, Multi-level visualization tasks, Storage-computingrendering, Collaborative scheduling, Flexible allocation strategy, Adaptive optimization mechanism

\begin{abstract}
:
To address the multi-modal spatio-temporal data efficient scheduling problem of the diverse and highly concurrent visualization applications in cloud-edge-terminal environment, this paper systematically studies the cloud-edge-terminal integrated scheduling model of multi-level visualization tasks of multi-modal spatio-temporal data. By accurately defining the hierarchical semantic mapping relationship between the diverse visual application requirements of different terminals and scheduling tasks, we propose a multi-level task-driven cloud-edge-terminal multi-granularity storage-computing-rendering resource collaborative scheduling method. Based on the workflow, the flexible allocation strategy of cloud-edge-terminal scheduling service chain that consider the characteristics of spatio-temporal task is constructed. Finally, we established a cloud-edge-terminal scheduling adaptive optimization mechanism based on the service quality evaluation model, and developed a prototype system. Experiments are conducted with the urban construction and construction management, the results show that the new method breaks through the bottleneck of traditional spatiotemporal data visualization scheduling, and it can provide theoretical and methodological support for the visualization and scheduling of spatio-temporal big data.
\end{abstract}

\section{INTRODUCTION}

The development of social network, sensor network, Internet of Things (IoT) and their multi-layer coupling data collection and recording technology makes the acquired spatio-temporal data of social world, computer world and material world have multimodal characteristics. These multi-modal spatio-temporal data describe the holographic feature information of multigranularity spatio-temporal objects in the full life cycle, such as the position, geometry, behavior, and semantic relationship of the multi-granular spatio-temporal objects. Multi-modal spatiotemporal data generally includes photorealistic fine geometry, textures and materials, video, photos and non-photorealistic computing and simulation result data, abstract expression symbols (Valencia et al., 2015). These unstructured and sparse data make a huge challenges for storage, computing, and rendering (Wang et al., 2013; Yang et al., 2013). In order to satisfy the requirements of high concurrency and high real-time during multimodal spatio-temporal data visualization, a hybrid architecture integrating cloud computing, edge computing and multi-terminal (cloud-edge-terminal) can be an effective solution. Although the hybrid architecture effectively reduce network delay and improve response speed by accelerating the computing with the edge equipment near the user terminal, it may also greatly increase complexity and instability of resource scheduling (Shi et al., 2016; Satyanarayanan 2017). The massive, high-dimensional and dynamic characteristics of multi-modal spatio-temporal data lead to visual content and scene dynamic changes for the visualization application (Peters et al., 2017). Diverse visualization tasks are interwoven, highly concurrent and relies heavily on storage, computing and visualization resources (Yang et al., 2011; Wong et al., 2012).

The core problem for multi-level and diverse visual scheduling of multi-modal spatio-temporal data has been studied by the researchers, include null data visualization task model, dynamic spatio-temporal data scheduling, resource scheduling in cloud environment and so on. According to the decomposition of the visualization task and the association between tasks, the spatiotemporal data visualization task model can be divided into low-level, high-level and multi-level three categories (Amar et al., 2005; Tory et al., 2004; Brehmer et al., 2013). Pike (2009) has focused on data processing methods in visualization and analysis applications, which typical contents include browsing, identification, coding, abstraction, figuration and filtering (Ward et al., 2010). The description of the high-level visualization task model mainly focuses on the differences in the visual analysis stage, and its typical contents include data collection, data browsing, and data analysis and so on (Nazemi, 2016). Andrienko (2003) and Nusrat (2015) have shown that the multi-level visualization task model can uniformly describe the coupling relationship between tasks, which typical contents include visualization purpose, visualization method, visualization content, visualization time, visualization space, and visualization user. However, the existing visualization task model is mainly data-centric, and it is difficult to meet the diverse and highly concurrent needs of visualization tasks.

\footnotetext{
* Corresponding author: Xiaoming Li, lixming@szu.edu.cn
} 
Efficient spatio-temporal data dynamic scheduling method is the key to realizing high performance large-scale spatiotemporal data visualization and other applications. For largescale terrain rendering, mainly by constructing a terrain pyramid and dynamically scheduling terrain tiles of different resolutions to achieve high efficient and high fidelity terrain scheduling and visualization (Kang et al., 2010; Strugar et al., 2009). At the same time, a multi-level of detail model has been construct to solve the visualization of the urban 3D model with uneven spatial distribution, and the Out-of-Core Rendering technology is used for scheduling and visualization (Mason et al., 2001; Li et al., 2011; Biljecki et al., 2014). The performance of existing visualization and scheduling methods of spatiotemporal scenes mainly rely on the spatial index, dynamic data scheduling and data simplification and other optimization methods (Chen et al., 2015; Maglo et al., 2015; Petring et al., 2013). However, the most of existing visualization and scheduling methods are mainly focused on graphical algorithms, which lacks collaborative scheduling of visualization system resources. Generally, the existing spatio-temporal data scheduling schemes are mainly based on the optimization of graphics algorithms, which can take into account the diversified network environment and the differences in visualization equipment to a certain extent. However, the scene organization method determines that the spatio-temporal scene data needs to be processed according to a specific organization form, resulting in a fixed scene representation. At the same time, this scheduling method optimized by graphics can no longer meet the needs of diversified visualization for diverse computing platforms and access terminals (Evans et al., 2014; Evangelidis et al., 2014; Hähnle et al., 2015).

In the cloud environment, storage, computing, and rendering resources are integrated into a huge virtual resource pool. According to the demand for resources, multiple computing nodes, which can be instantiated in the resource pool. Then, tasks such as data analysis and computing, and scene rendering are distributed and run on different computing nodes in the form of services or applications. How to allocate tasks with different resource requirements to various computing nodes and the dynamic expansion of computing nodes have become a research hotspot in resource scheduling in the cloud environment, thereby improve load balancing and maximize task execution efficiency with the premise of satisfying user service quality. There are two types of resource scheduling in the cloud environment: traditional resource scheduling and heuristic scheduling. Although the traditional resource scheduling method has a simple algorithm and low computing cost, its performance and flexibility are not high enough, which unsuitable for complex network environments and task scheduling with complex relationships between subtasks. Although the heuristic scheduling algorithm is an effective method to find the multi-objective optimal solution, there are still some defects. As the online video, augmented reality and virtual reality services spawned by the era of big data, more strict functions and performance requirements for caching, delay, policy control are put forward. Although the cloud computing has logical resource concentration advantage, rely on heavy cloud computing that is far away from the user terminal will inevitably lead to a bottleneck effect. For the diverse and high-concurrency and high real-time application requirements of large-scale spatio-temporal data, optimizing the collaborative scheduling algorithm of resources under the cloud-edge-terminal hybrid architecture becomes very important (Chen et al., 2016; Shi et al., 2016).
In this paper, a new cloud-edge-terminal resources collaborative scheduling model for multi-level visualization of large-scale multi-modal data is proposed. Firstly, we systematically study the integrated scheduling model of cloud-edge-terminal for multimodal spatiotemporal data multi-level visualization tasks. This model accurately depicts the hierarchical semantic mapping relationships between the diversified visualization application requirements of different terminals and scheduling tasks. Then, a multi-granularity storage-computing-rendering resources collaboration method based on multi-level visualization task-driven is proposed. Finally, an adaptive taskdriven scheduling engine system is developed.

The remainder of this paper is organized as follows. Section 2 describes the system and method. Section 3 presents the related experiments. Section 4 summarizes this work and discusses future research directions.

\section{METHOD}

\subsection{Cloud-edge-terminal resources collaborative scheduling model}

In general, there are three characteristics of multi-modal spatiotemporal data in cloud-edge-terminal application environment, stored in the cloud center, accelerated computing at the edge server and used in multi-terminal applications. As the traditional spatio-temporal data visualization scheduling mainly foucs on the single spatio-temporal scene display task, it cannot meet the needs of multi-terminal diversified visualization applications. Aiming at the limitation, a cloud-edge-terminal integrated scheduling model for multi-modal spatio-temporal data multi-level visualization tasks is proposed. The integrated scheduling model is shown in Figure. 1. There are three steps involved in the cloud-edge-terminal resources collaborative scheduling model.

2.1.1 Construction of hierarchical semantic mapping relationship between the multi-level visualization tasks and scheduling tasks

Based on the four dimensions of spatio-temporal data ( Data), analytical computing model ( Model ), human-computer interaction (Interaction) and rendering ( Render) included in the visualization, the multi-modal spatio-temporal data visualization task can be denoted as:

$$
\text { VTask }=\langle\text { Data, } \text { Model, } \text { Interaction, } \text { Render }\rangle
$$

where VTask = multi-modal spatio-temporal data visualization task

By constructing data browsing, data analysis and knowledge acquisition models in the multi-modal spatio-temporal data visualization requirements, the multi-modal spatio-temporal data visualization task is described in three levels: display visualization task which only includes data scheduling and scene rendering task, analytical visualization task and exploratory visualization task. Analytical visualization task mainly includes data scheduling, computing analysis and scene rendering task, whereas exploratory visualization task includes data scheduling, computing analysis, interactive computing and scene rendering task.

$$
\begin{aligned}
& \text { VTask }_{V}=\langle\text { Data }, \text { Render }\rangle \\
& \text { VTask }_{A}=\langle\text { Data }, \text { Model }, \text { Render }\rangle \\
& \text { VTask }_{E}=\langle\text { Data }, \text { Model }, \text { Interaction, Render }\rangle
\end{aligned}
$$

where $\quad$ VTask $_{V}=$ display visualization task 


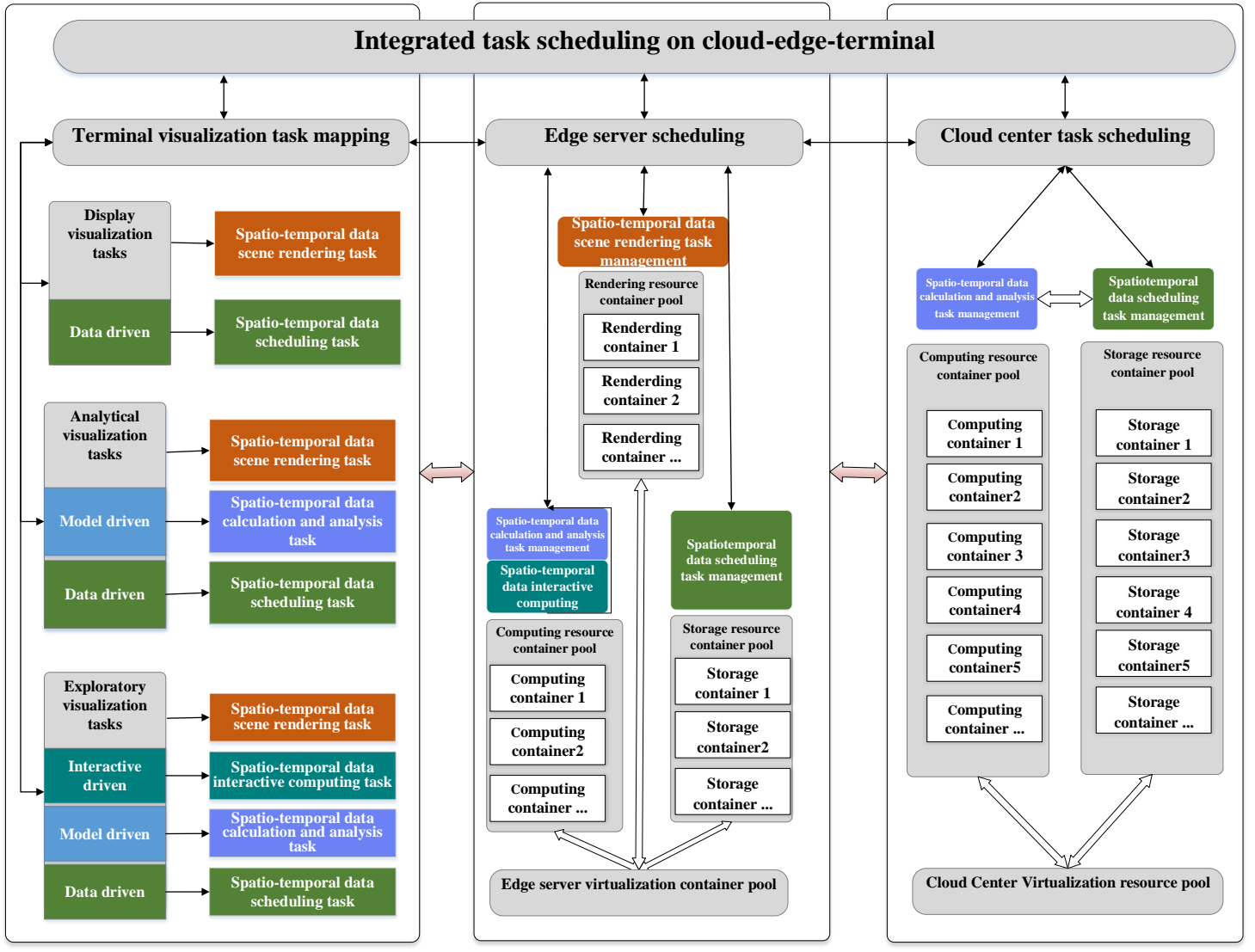

Figure. 1 Integrated scheduling model of cloud-edge- terminal for multi-level visualization tasks

$$
\begin{aligned}
& \operatorname{VTask}_{A}=\text { analytical visualization task } \\
& \operatorname{VTask}_{E}=\text { exploratory visualization task }
\end{aligned}
$$

According to the three levels of content contained in the multilevel visualization task model, the multi-level visualization task model can be described as:

$$
\text { VTaskModel }=\left\langle\text { VTask }_{V}, \text { VTask }_{A}, \text { VTask }_{E}\right\rangle
$$

Then, the system-level resources and spatio-temporal data scene optimization content on which the display, analytical, and exploratory visualization tasks in the multi-level visualization task model are defined. In order to realize the hierarchical semantic mapping of the multi-level visualization task to the scheduling task, we establish the dependency relationship between the multi-level visualization task and system resources and data content. The multi-level visualization task can be converted into multi-level scheduling task that include data scheduling task, computing analysis task, interactive computing task, and rendering task.

$$
\text { STaskModel }=\left\langle\text { STask }_{D}, \text { STask }_{A}, \text { STask }_{I}, \text { STask }_{V}\right\rangle
$$

where $\quad$ STaskModel $=$ multi-level scheduling task

STask $_{D}=$ data scheduling task

STask $_{A}=$ computing analysis task

STask $_{I}=$ interactive computing task

STask $_{V}=$ rendering task

At the same time, display visualization task can be mapped to spatio-temporal data scheduling task and spatio-temporal data scene rendering task. Analytical visualization task add a spatiotemporal data computing and analysis task mapping compared to display visualization task. Compared to analytical visualization task, the exploratory visualization task further includes the spatio-temporal data interactive computing task mapping.

$$
\begin{aligned}
& \operatorname{VTask}_{V}=\left\langle\operatorname{STask}_{D}, \text { STask }_{V}\right\rangle \\
& \operatorname{VTask}_{A}=\left\langle\operatorname{STask}_{D}, \text { STask }_{A}, \text { STask }_{V}\right\rangle \\
& \operatorname{VTask}_{E}=\left\langle\operatorname{STask}_{D}, \text { STask }_{A}, \text { STask }_{I}, \text { STask }_{V}\right\rangle
\end{aligned}
$$

2.1.2 Fine-grained decomposition of scheduling tasks based on spatiotemporal semantic association

In order to reduce the complexity of the task and improve the balance and efficiency of the assignment of subsequent scheduled tasks, it is necessary to finely decompose the scheduling subtasks at different levels according to the spatiotemporal semantic association of multimodal spatiotemporal data. At the same time, consider decomposing the information interaction relationship between scheduling subtasks, constructing a combination of different levels of scheduling subtasks and nested task sets. Then, in order to reduce the degree of information interaction between the decomposed subtasks and improve the matching efficiency and accuracy of tasks and resources, the decomposed subtasks are grouped. Two factors should be considered when grouping, subtasks with high information interaction are classified into the same group as much as possible, and the workload of each group is balanced. Specifically, spatio-temporal data scheduling tasks can be subdivided into sub-tasks such as basic scene data scheduling, dynamic scene data scheduling, data scheduling required for computing analysis, and data scheduling required for interactive computing. The spatio-temporal data scene rendering tasks can be subdivided into sub-tasks such as basic scene rendering, dynamic scene rendering, scene interactive rendering, etc. For space-time data computing and analysis 
tasks and interactive computing tasks, fine-grained decomposition is required according to data content and computing analysis content. The data required for computing generates the corresponding data scheduling task, and the result generated by the computing is converted into the corresponding scene rendering task.

\subsubsection{Cloud-edge-terminal integrated scheduling model} and workflow

According to the characteristics of multi-modal spatio-temporal data in the cloud-edge-terminal application environment, the storage resources, computing resources and rendering resources on the cloud side can be described as:

$$
\text { Resource }=\left\langle\operatorname{Res}_{D}, \operatorname{Res}_{C}, \operatorname{Res}_{V}\right\rangle
$$

where $\operatorname{Res}_{D}=$ storage resources

$\operatorname{Res}_{C}=$ computing resources

$\operatorname{Res}_{V}=$ rendering resources

The storage resources mainly perform data scheduling tasks, whereas computing resources mainly perform computing analysis and interactive computing tasks. The rendering resources mainly perform rendering tasks. By building a task manager for integrated scheduling on the cloud-edge-terminal, the computing, storage and rendering resources on the cloudedge-terminal are scheduled in an integrated manner. The cloud-edge-terminal integrated scheduling model can be described as:

ResourceModel $=\langle$ CloudResourceModel,

EdgeResourceModel,TerminalResourceModel $\rangle$

where CloudResourceModel $=$ Cloud centre

EdgeResourceModel = Edge server

TerminalResourceModel $=$ User terminal resources

The cloud centre mainly provides computing and storage resource functions, and performs computing analysis, interactive computing and data scheduling task. The edge server mainly provides computing, storage and rendering resource functions, and performs computing analysis, interactive computing, data scheduling and rendering tasks. The user terminal resources will vary greatly according to the differences between high-performance desktop terminals, lightweight mobile terminals, and virtual-augmented reality devices. After all, the cloud-edge-terminal integrated scheduling model can achieve a complete description of the storage, computing and rendering resources of the cloud centre, edge server and user terminal resources. At the same time, the cloud-edge-terminal integration model establishes the corresponding relationship between these resources and different levels of scheduling tasks. Through the mapping relationship between these scheduling tasks and multi-level visualization tasks, the cloud-edgeterminal integration model realizes the construction of the dependency relationship between different levels of visualization tasks and cloud-edge-terminal storage, computing and rendering resources.

2.2 Multi-granularity resources collaboration method

According to the characteristics and requirements of different levels of tasks, a multi-level task-driven cloud-edge-terminal multi-granularity storage and drawing resource collaborative scheduling method is designed. The new method enables the cloud-edge-terminal storage and drawing resources to collaboratively handle multi-level scheduling tasks such as spatio-temporal data scheduling, spatio-temporal data computing analysis, spatio-temporal data interactive computing, and spatio-temporal scene drawing. There are three steps involved in the multi-granularity storage-computing-rendering resources collaboration method.

2.2.1 Construction of workflow for storage-computingrendering resources collaboration based on spatio-temporal semantics and distribution characteristics

First of all, the infrastructure in cloud computing technology is used to gather the storage, computing and rendering resources distributed in the system to form a resource pool that can be allocated on demand. Then according to the spatio-temporal data reading and writing, analysis model and scene optimization to deal with the demand for resources. Multi-granular storage, computing and rendering services that can be dynamically configured and instantiated are published and managed by microservice architecture. Then, according to the requirements of spatio-temporal scene data and the demand for the operation of storage, computing and rendering resources, constructing a workflow for storage-computing-rendering resources collaboration based on spatio-temporal semantics and distribution characteristics. In addition, according to the spatiotemporal semantic association relationship and distributed storage characteristics of multimodal spatiotemporal data is handled. Task workflow include data scheduling tasks, scene rendering tasks, computing analysis tasks and interactive computing tasks and so on. Finally, establish a dynamic mapping relationship between the storage-computing-rendering resource collaborative scheduling workflow of cloud-edgeterminal and the multi-granularity storage-computing-rendering services of cloud-edge-terminal.

2.2.2 Dynamic construction of cloud-edge-terminal collaborative scheduling service chain based on workflow

The collaborative scheduling workflow for storage-computingrendering resource on the cloud side provides a template process for multi-level scheduling tasks on the cloud-edgeterminal, such as task scheduling, resource allocation, and status monitoring. Based on the collaborative scheduling workflow at the cloud-edge-terminal, the multi-granularity storagecomputing-rendering service can be constructed as a collaborative scheduling service chain according to demand, which shown in Figure. 2. First, build a service chain with different granularity of storage-computing-rendering services as basic units, and these service units can be dynamically combined in the service chain. Then, according to the storage, computing and drawing task requirements involved in the scheduling task, the scheduling service chain combines the service units of multi-granular storage-computing-rendering services. Finally, the scheduling tasks of the scheduling service chain are automatically executed based on the scheduling workflow.

2.2.3 Flexible allocation strategy of scheduling service chain considering spatio-temporal characteristics

The cloud-edge-terminal scheduling service chain not only needs to dynamically combine service units according to the requirements of scheduling tasks, but more importantly, it should provide a flexible service allocation mechanism. The flexible service allocation mechanism can allocate resources according to the scheduling tasks and cloud-edge-terminal resource load requirements, so as to quickly respond to various scheduling task requests of each user terminal. At the same time, due to continuous access to real-time data, interactive operation of spatio-temporal scenes and other reasons, changes in spatiotemporal scenes may cause mutations in scheduling tasks. Therefore, it is necessary to carry out special targeted task scheduling and resource allocation optimization for the scheduling service chain based on the characteristics of spatiotemporal tasks. Furthermore, we stablished a flexible allocation 
strategy of cloud-edge-terminal scheduling service chain which

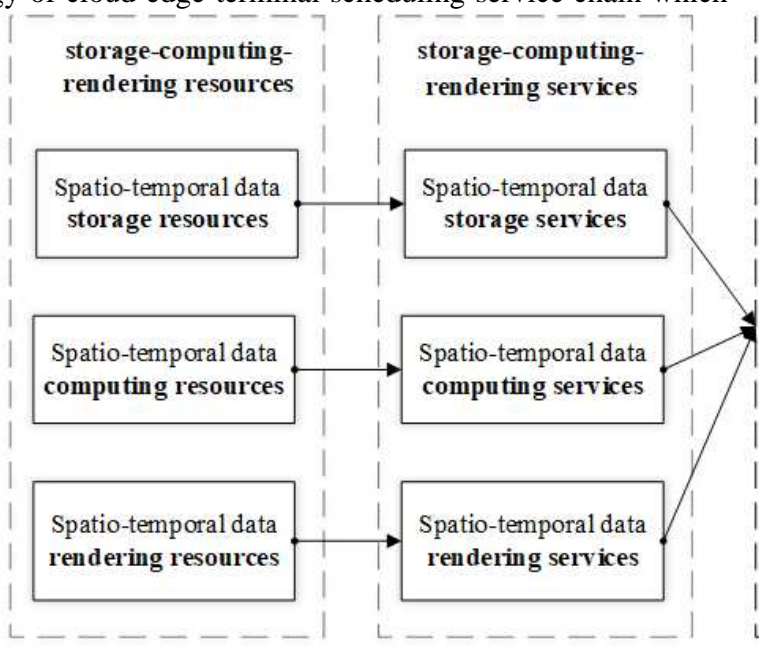

Figure. 2 Task driven multi-granularity storage-computing-rendering resources collaborative scheduling mechanism considering the characteristic of spatio-temporal task

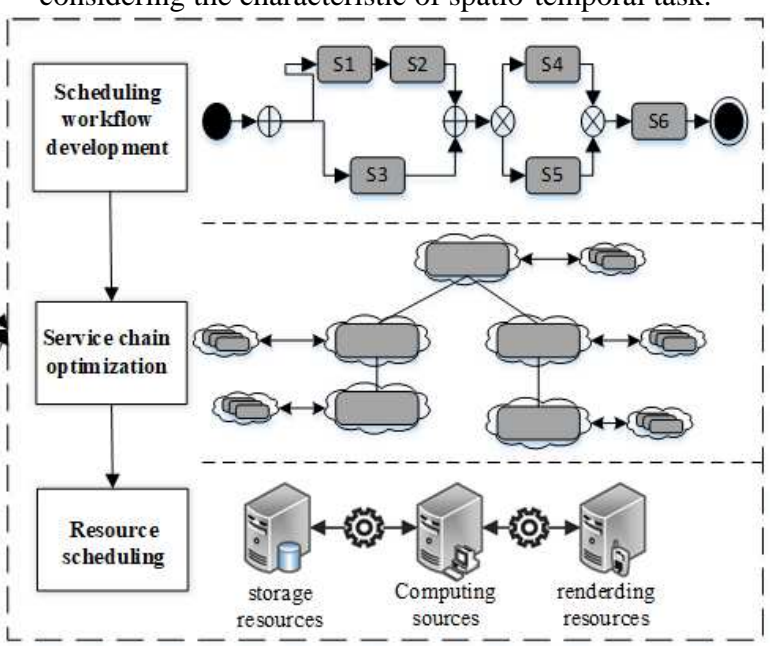

\section{EXPERIMENTAL ANALYSIS}

\subsection{Cloud-edge-terminal resources scheduling prototype system construction \\ collaborative}

Based on task-driven collaborative scheduling method of cloudedge-terminal multi-granularity storage-computing-rendering services, an adaptive and task-driven scheduling engine prototype system was developed. We built a distributed cloudedge-terminal hardware environment composed of diversified terminals (terminal), edge server cluster (edge), cloud center equipment (cloud) and network equipment for testing. A microservice architecture with high performance, high availability and high scalability was built to realize decentralized governance in a high scalable cloud environment. We use the 3D model data of Shenzhen to build the 3D city scene, and dynamically access the IOT sensor data to build a multimodal spatio-temporal dataset. Aiming at different user terminals such as high-performance desktop terminal, lightweight mobile terminal and virtual / augmented reality terminal, three levels of visualization are used to verify the effectiveness of the cloud side collaborative scheduling prototype system in this paper. The cloud-edge-terminal microservice architecture of collaborative scheduling engine prototype system is shown in Figure. 3.

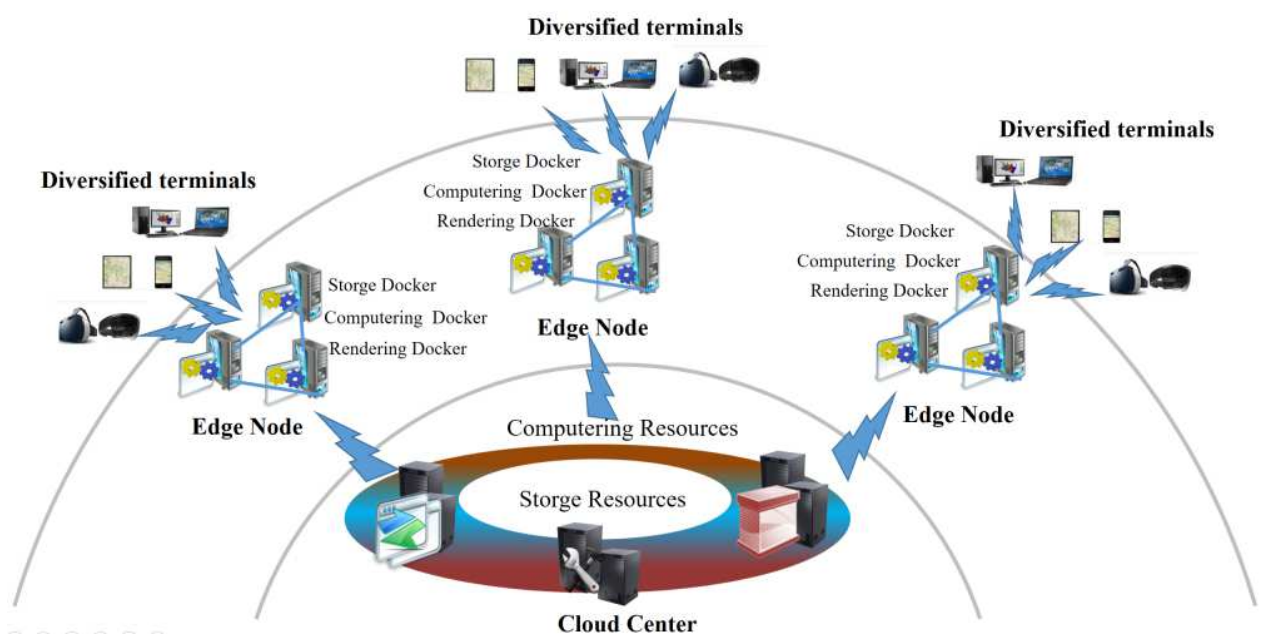

Figure. 2 The cloud-edge-terminal microservice architecture of prototype system

The traditional integrated GIS architecture has many problems, such as centralized deployment, high coupling between components, and difficult to maintain and continuously update. In this paper, the GIS microservice architecture is designed to realize decentralized deployment, loose coupling between services, decentralized governance in a highly scalable cloud environment, and significantly improve the agility and maintainability of multimodal spatiotemporal data visualization system iteration.

According to the idea of GIS microservice architecture, the traditional integrated service application architecture is decomposed into independent service clusters. Each service in the service cluster runs in its own process and uses a unified multilingual service description. Lightweight communication mechanism is used for data interaction between services and services. Thus, it can ensure the mutual invocation of heterogeneous services and support the independent deployment of services. At the same time, through the service registry to manage and track the call dependency relationship between services, we can realize the efficient management of multi granularity services with complex relationship.

The container management of microservices is shown in figure 4. Container technology is used to package the service and its dependent software running environment as image. Each service is packaged through docker container, and unified service arrangement and management are carried out through 
kubernetes. At the same time, isito is used to build and manage the service mesh composed of mutual calls between microservices.

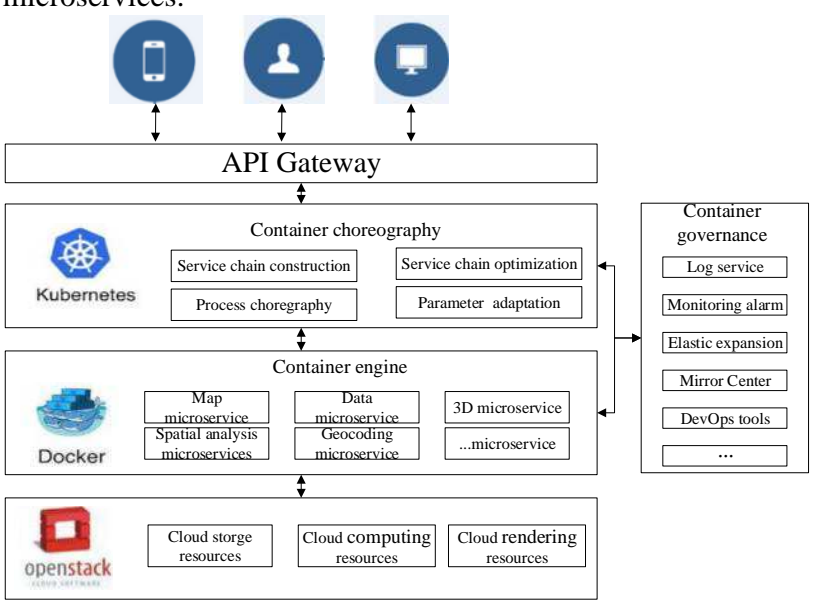

Figure. 4 Container management of microservices

\subsection{Experimental application}

In this paper, a cloud side distributed hardware test environment is built, which is composed of diversified terminals (end), edge

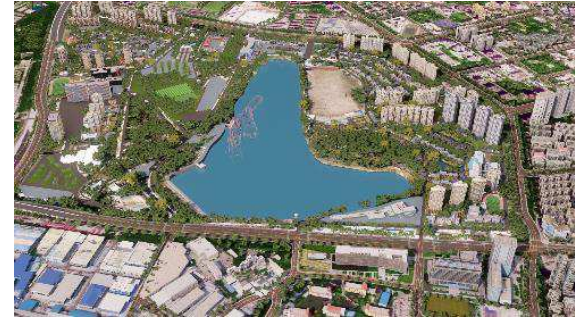

(a) Large-scale 3D city scene visualization

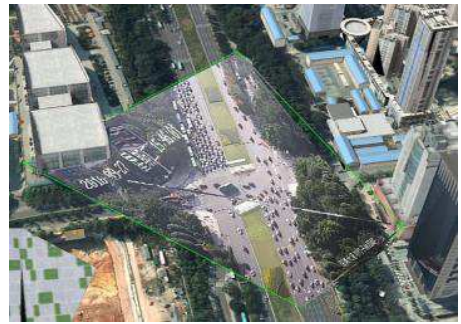

(b) City traffic flow simulation server cluster (edge), cloud center equipment (cloud) and network equipment. Then, we simulate multi-user concurrency in multi-terminal to build a large-scale user high concurrency experimental test environment. At last, we test visualization capabilities for different terminals, such as high-performance desktop terminals, lightweight mobile terminals, virtual / augmented reality terminals, etc. Experiments show that the system can increase the number of service instances and improve the system throughput through horizontal expansion when the resource conditions allow. For different terminals, the system has the ability to store, compute and draw resources adaptively

In order to meet the needs of smart city construction, three typical cases are validated and analyzed: the visualization of large-scale 3D city scene visualization, the visualization of dynamic city traffic flow simulation, and the exploration of indoor fire evacuation. The result shows that the proposed framework can effectively provide the multi-level visual application of multi-domain in the whole life cycle of urban overview, planning, operation, maintenance, and emergency disaster response. The spatio-temporal multi-level visualization application cases are shown in Figure. 5.

Figure. 5 The spatio-temporal multi-level visualization application cases

\section{CONCLUSION}

Nowadays, the traditional spatio-temporal data visualization scheduling method of has been difficult to meet the requirements of diverse visualization applications of different terminals, as it is usually used for a single display task of desktop terminals. Due to the limitations of the traditional methods that lacks cloud-edge-terminal integrated scheduling model for multi-level visualization tasks, the self-adaptive scheduling mechanism of efficient collaborative scheduling for cloud-edge-terminal storage-computing-rendering resources is further studied in this paper.

Based on the cloud-edge-terminal hybrid architecture, this paper systematically studies thecloud-edge-terminal resource collaborative scheduling method for multi-level visualization tasks. First, the dependency relationship between multi-level and diverse visualization requirements and data and system resources is described based on the multi-level visualization task model of multi-modal spatio-temporal data. Then, a taskdriven cloud-edge-terminal multi-granularity storage and drawing resource collaborative service method is proposed, which efficiently coordinate the scheduling of cloud-edgeterminal storage-computing-rendering resources and realizes the rapid response of multi-level visualization analysis application. Experiments are conducted with the urban construction and construction management, the results show that the new method breaks through the bottleneck of traditional spatiotemporal data visualization scheduling, and it can provide theoretical and methodological support for the visualization and scheduling of spatio-temporal big data.

\section{ACKNOWLEDGEMENTS}

Acknowledgements of support for the National Natural Science Foundation of China (Grant No. 41971341), General Project of the National Natural Science Foundation of Guangdong Province (Grant No. 2019A1515010748, 2019A1515011872) and New Teacher Research Project of Shenzhen University (Grant No. 2019056).

\section{REFERENCES}

Amar, R., Eagan, J., Stasko, J., 2005. Low-level components of analytic activity in information visualization. In: IEEE Symposium on Information Visualization, Minneapolis, MN, USA, pp. 111-117. 
Andrienko, N., Andrienko, G., Gatalsky, P., 2003. Exploratory spatio-temporal visualization: an analytical review. Journal of Visual Languages \& Computing, 14(6), pp. 503-541.

Biljecki, F., Ledoux, H., Stoter, J., et al., 2014. Formalisation of the level of detail in 3D city modelling. Computers, Environment and Urban Systems, 48, pp. 1-15.

Brehmer, M., Munzner, T. A., 2013. multi-level typology of abstract visualization tasks. IEEE Transactions on Visualization and Computer Graphics, 19(12), pp. 2376-2385.

Chen, J., Li, M., Li, J., 2015. An improved texture-related vertex clustering algorithm for model simplification. Computers \& Geosciences, 83, pp. 37-45.

Chen, J., Li, J., Li, M., 2016. Progressive Visualization of Complex 3D Models Over the Internet. Transactions in GIS, 20(6), pp. 887-902.

Evangelidis, K., Ntouros, K., Makridis, S., et al., 2014. Geospatial services in the Cloud. Computers \& Geosciences, 63, pp. 116-122.

Evans, A., Romeo, M., Bahrehmand, A., et al., 2014. 3D graphics on the web: A survey. Computers \& Graphics, 41, pp. 43-61.

Hähnle, R., Johnsen, E. B., 2015. Resource-aware applications for the cloud. IEEE Computer, 48(6), pp. 72-75.

Kang, L., Xu, J., Yang, C., et al., 2010. An efficient simplification and real-time rendering algorithm for large-scale terrain. International Journal of Computer Applications in Technology, 38(1-3), pp.106-112.

Li, J., Wu, H., Yang, C., et al., 2011. Visualizing dynamic geosciences phenomena using an octree-based view-dependent LOD strategy within virtual globes. Computers \& geosciences, 7(9), pp. 1295-1302.

Maglo, A., Lavoué, G., Dupont, F., et al., 2015. 3d mesh compression: Survey, comparisons, and emerging trends. ACM Computing Surveys (CSUR), 47(3), pp. 44.

Mason, A. E. W., Blake, E. H., 2001. A graphical representation of the state spaces of hierarchical level-of-detail scene descriptions. IEEE Transactions on Visualization and Computer Graphics, 7(1), pp. 70-75.

Nazemi, K., 2016. Adaptive semantics visualization. Springer.

Nusrat, S., Kobourov, S., 2015. Task taxonomy for cartograms. In: 17th IEEE Eurographics Conference on Visualization, pp.11-17.

Petring, R., Eikel, B., Jähn, C., et al., 2013. Real-time 3D rendering of heterogeneous scenes. In: International Symposium on Visual Computing, Springer, Berlin, Heidelberg, pp. 448-458.

Peters, S., Jahnke, M., Murphy, C. E., et al., 2017. Advances in 3 D Geoinformation. Springer, Cham, pp. 207-230.

Pike, W. A., Stasko, J., Chang, R., et al., 2009. The science of interaction. Information Visualization, 8(4), pp. 263-274.

Satyanarayanan, M., 2017. The emergence of edge computing Compute, 50(1), pp. 30-39.
Shi, W., Cao, J., Zhang, Q., et al., 2016. Edge computing: Vision and challenges. IEEE Internet of Things Journal, 3(5), pp. 637-646.

Strugar, F., 2009. Continuous distance-dependent level of detail for rendering heightmaps. Journal of graphics, GPU, and game tools, 14(4), pp. 57-74.

Tory, M., Moller, T., 2004. Rethinking visualization: A highlevel taxonomy. In: IEEE Symposium on Information Visualization, Austin, Texas, USA, pp. 151-158.

Valencia, J., Muñoz-Nieto, A., Rodriguez-Gonzalvez, P., 2015. Virtual Modeling for Cities of the Future. State-Of-The Art and Future Challenges. In: The International Archives of the Photogrammetry, Remote Sensing and Spatial Information Sciences, Avila, Spain, Volume XL-5, Part W4, pp. 179-185.

Wang, S., Anselin, L., Bhaduri, B., et al., 2013. CyberGIS software: a synthetic review and integration roadmap. International Journal of Geographical Information Science, 27(11), pp. 2122-2145.

Ward, M. O., Grinstein, G., Keim, D., 2010. Interactive data visualization: foundations, techniques, and applications. CRC Press.

Wong, P. C., Shen, H. W., Johnson, C. R., et al., 2012. The top 10 challenges in extreme-scale visual analytics. IEEE computer graphics and applications, 32(4), pp. 63-67.

Yang, C., Goodchild, M., Huang, Q., et al., 2011. Spatial cloud computing: how can the geospatial sciences use and help shape cloud computing?. International Journal of Digital Earth, 4(4), pp. 305-329.

Yang, C., Xu, Y., Nebert, D., 2013. Redefining the possibility of digital Earth and geosciences with spatial cloud computing. International Journal of Digital Earth, 6(4), pp. 297-312. 